\title{
Comparação entre as necessidades energéticas prescritas e adminis- tradas a pacientes em terapia nutricional enteral
}

\section{Comparison of the energy needs a prescribed and administered enteral nutrition therapy in patients}

\author{
Claudia R. P. Detregiachi' ${ }^{1}$ Karina R. Quesada², Dayane E. Marques ${ }^{3}$
}

\begin{abstract}
RESUMO
Objetivo: comparar a adequação, prescrição e oferta energética da terapia de nutrição enteral em pacientes hospitalizados. Metodologia: foi realizado um levantamento retrospectivo do protocolo de evolução de TNE de 59 pacientes hospitalizados em um hospital geral da cidade de Marília/SP/BR. Os dados coletados incluíram: sexo, idade, diagnóstico clínico, dados antropométricos e dietéticos referentes a fórmula dietética prescrita, via e método de administração da nutrição enteral, o volume diário prescrito e o administrado da mesma, valor diário de energia prescrito e ofertado da dieta, assim como as intercorrências. Para avaliar o estado nutricional dos pacientes utilizou-se o índice de massa corporal (IMC), dobra cutânea tricipital (DCT) e circunferência muscular do braço (CMB). A adequação da TNE foi avaliada com base na necessidade energética diária comparada com o valor energético médio recebido diariamente durante o período de uso da TNE. Resultados: Quanto ao IMC, a média encontrada entre os pacientes avaliados foi de $21,4 \mathrm{~kg} / \mathrm{m}^{2}$, não havendo diferença entre os sexos. Os dados da DCT e CMB apresentaram diferença entre os sexos $(p<0,05)$ e nos sugerem maior comprometimento da massa muscular em relação ao tecido adiposo. A necessidade energética média foi de $1642 \mathrm{kcal} /$ dia. Entretanto a média de energia prescrita foi de $1045 \mathrm{kcal} / \mathrm{dia}$ e a ofertada foi de $1035 \mathrm{Kcal} / \mathrm{dia}$. Verificou-se uma diferença significativa entre a necessidade e a oferta energética por meio da nutrição enteral $(p<0,05)$ assim como entre ao valor energético necessário e o prescrito $(p<0,05)$, ficando ambos aquém da necessidade. Não houve diferença significativa $(p>0,05)$ nos parâmetros de oferta energéti$\mathrm{ca}$, volumes de NE prescrito e administrado entre os pacientes eutróficos, com baixo peso ou com excesso de peso. Conclusão: Os resultados deste estudo indicam que a prescrição e a oferta energética não foram baseadas nas necessidades destes pacientes, acarretando um déficit energético importante, que pode levar ao agravo do estado nutricional dos mesmos. Em pacientes hospitalizados em uso de TNE, a prescrição energética deve ser feita pelo profissional habilitado dentro da equipe multidisciplinar, tendo como base as necessidades de cada paciente.
\end{abstract}

Palavras-chave: Terapia nutricional. Nutrição enteral. Estado nutricional. Necessidades nutricionais. Hospitalização.

\footnotetext{
${ }^{1}$ Docente da Universidade Estadual Paulista - Unesp - Botucatu e da Universidade de Marília - UNIMAR. Pós-doutoranda - Universidade Estadual Paulista - Unesp - Botucatu, Brasil.

${ }^{2}$ Docente da Universidade de Marília - Unimar e Universidade Paulista - Unip - Assis, Brasil. Mestranda Alimentos e Nutrição: Ciências Nutricionais - Universidade Estadual Paulista - Unesp Araraquara, Brasil.

${ }^{3}$ Nutricionista. Aprimoranda de Nutrição Clínica - Faculdade de Medicina de Marília - FAMEMA-Marília, Brasil.
}

Correspondência: Claudia Rucco Penteado Detregiachi Rua Nassimen Mussi, 333 - Jardim Itaipú 17519-570 - Marília/SP - Brasil

Fone: (14) 8144-1019.

E-mail: claudiarucco@ibb.unesp.br / claudiarucco@flash.tv.br

Artigo recebido em 09/08/2010 Aprovado para publicação em 16/03/2011 


\section{Introdução}

A terapia nutricional enteral (TNE) constitui um conjunto de procedimentos terapêuticos adotados para manutenção ou recuperação do estado nutricional do paciente por meio de nutrição enteral (NE). ${ }^{1}$ Este mesmo documento define NE como "alimento para fins especiais, com ingestão controlada de nutrientes,... especialmente formulada e elaborada para uso por sondas ou via oral...". Com base em tais definições, compreende-se que a TNE é uma alternativa terapêutica que viabiliza a manutenção do estado nutricional em pacientes com impossibilidade parcial ou total de manter a via oral como rota de alimentação, devendo ser adotada sempre que o trato gastrointestinal estiver funcionante. ${ }^{2}$ Ademais, de acordo com Baxter e Waitzberg, ${ }^{3}$ há indicação desta via de alimentação a qualquer indivíduo sem condições de atender ao menos $60 \%$ de suas necessidades nutricionais, voluntariamente, por meio da via oral.

Na impossibilidade ou dificuldade do uso da via oral, a TNE é vista como uma ferramenta nutricional prioritária comparada à nutrição parenteral por apresentar vantagens fisiológicas, metabólicas, de segurança e de melhor relação custo/benefício. ${ }^{4} \mathrm{~A}$ oferta de nutrientes por via enteral mantém a estrutura e a microflora intestinais normais, além de otimizar o sistema imunológico intestinal e manter a homeostase. Ressalta-se ainda associação significativa com a redução de incidência de complicações infecciosas, decréscimo na taxa metabólica e melhora do balanço nitrogenado em pacientes no pós-operatório. ${ }^{5}$

Entretanto, a prescrição de TNE é um processo complexo que implica conhecimento clínico (avaliação de doença de base) e nutricional. ${ }^{6}$ Com um esforço multidisciplinar, deve-se buscar uma prescrição dietética adequada assim como a infusão da NE em doses plenas, com vista a alcançar os benefícios que a mesma pode proporcionar. Tão importante quanto a prescrição adequada da TNE é a certeza de que o paciente estará recebendo a dieta prescrita. $^{7}$

A desnutrição configura-se como um dos principais fatores responsáveis pelos maiores índices de mortalidade, cicatrização mais lenta de feridas, prolongamento da internação hospitalar, aumento das complicações, menor rotatividade de leitos e, consequentemente, maior custo no tratamento de doentes internados. ${ }^{8-11}$ Prevenir o comprometimento nutricional de pacientes hospitalizados deve ser foco da equipe de saúde.
Entretanto, apesar da reconhecida importância da adequada ingestão de nutrientes e energia como meio de prevenir a desnutrição, pacientes sob TNE frequentemente recebem um valor energético inferior às suas necessidades. ${ }^{7,12,13}$

Os fatores que impedem o adequado aporte nutricional enteral incluem os relacionados à intolerância da dieta (vômitos, diarréia, resíduo gástrico, distensão abdominal), os associados às práticas de rotina de enfermagem (manipulação do paciente, administração de medicamentos) e outras rotinas (procedimentos, exames). ${ }^{14}$

Considerando que a terapia nutricional precoce e adequada é um importante fator na promoção da saúde, diminuição do estresse fisiológico e manutenção da imunidade, avaliar a eficácia desse tratamento torna-se fundamental na prática clínica. ${ }^{15}$

Diante do exposto, o objetivo deste estudo foi comparar a adequação, prescrição e oferta energética da terapia nutricional enteral em pacientes hospitalizados.

\section{Metodologia}

Foi realizado um levantamento retrospectivo do protocolo de evolução de TNE de 59 pacientes hospitalizados em um hospital geral da cidade de Marília/ SP/BR, referente ao período de fevereiro a maio de 2009, após a aprovação pelo Comitê de Ética da Universidade de Marília - UNIMAR.

Os critérios de inclusão foram: idade igual ou superior a 18 anos e uso exclusivo de terapia enteral por pelo menos 72 horas.

Os dados foram coletados na planilha de evolução de TNE, incluindo: sexo, idade, diagnóstico clínico, dados antropométricos (peso, estatura, dobras cutâneas e circunferências braquial e muscular do braço) e dietéticos (fórmula dietética prescrita, via e método de administração da nutrição enteral, o volume diário prescrito e o administrado da mesma, valor diário de energia prescrito e ofertado da dieta), assim como as intercorrências justificadas nos casos de suspensão da oferta da nutrição enteral. No momento em que planilha de evolução de TNE indicasse a introdução de outra via de alimentação, oral ou parenteral, associada à nutrição enteral (NE), ou a suspensão da TNE ou ainda alta hospitalar ou óbito, sua avaliação era encerrada, determinando o tempo de avaliação de cada paciente.

Para avaliar o estado nutricional dos pacientes 
utilizou-se o índice de massa corporal (IMC), dobra cutânea triciptal (DCT) e circunferência muscular do braço (CMB). Para classificação do estado nutricional a partir do IMC utilizou-se os padrões propostos pela World Health Organization ${ }^{16}$ para adultos e, para pacientes acima de 60 anos, adotou-se os critérios de Lipschitz. ${ }^{17}$ A DCT e a CMB, tanto de adultos como idosos, foram avaliadas segundo os parâmetros propostos por Frisancho18 e classificadas quanto à adequação proposta por Blackburn \& Thornton ${ }^{19}$ nos quais os pontos de corte adotados para normalidade foram de 90 a $110 \%$ de adequação comparando com a medida no percentil . ${ }^{50}$

Os dados antropométricos foram coletados nas primeiras 24 horas do início da TNE, ou no primeiro dia útil após a introdução da TNE em casos de finais de semana. Portanto entre o $1^{\circ}$ e $3^{\circ}$ dia após a implementação da TNE.

A adequação da TNE foi avaliada com base na necessidade energética diária comparada com a média de valores energéticos prescritos e recebidos diariamente durante o período de uso da TNE. Para cálculo do gasto energético basal (GEB) foram utilizadas as equações propostas por Harris e Benedict ${ }^{20}$ e para o gasto energético total (GET) optou-se pelos fatores de lesão e atividade propostos por Long et al. ${ }^{21}$, correspondentes ao paciente. Os valores energéticos prescritos e recebidos foram calculados pela média do volume diário prescrito e administrado de $\mathrm{NE}$, respectivamente, coletado na planilha de evolução da TNE a partir do quarto dia de implementação da mesma e durante todo o período de avaliação do paciente. $\mathrm{O}$ resultado da análise desta adequação foi correlacio- nado com o diagnóstico nutricional dos pacientes segundo o IMC.

A opção por analisar a oferta da NE a partir do quarto dia foi baseada na expectativa, segundo Waitzberg ${ }^{4}$ de que nesse período o volume necessário para atingir necessidade energética fosse alcançado.

Os dados foram analisados utilizando o software BioEstat 5.0. Para tabulação dos valores encontrados foram utilizados média, desvio padrão, frequência relativa e, para análise estatística foi utilizado o teste de variância ANOVA, com valores considerados estatisticamente significativos se $\mathrm{p}<0,05$.

\section{Resultados}

A tabela 1 mostra o perfil geral da amostra estudada, dividida conforme o sexo. Foram avaliados 59 pacientes, sendo 30 do sexo feminino e 29 do masculino. Destes, $17 \%$ encontravam-se internados na Unidade de Terapia Intensiva (UTI) e os demais nas enfermarias. A média de idade foi de 69,3 $\pm 15,58$ anos. Diferentes doenças foram observadas nos pacientes avaliados, sendo elas a causa ou não da atual internação, tais como: doença cardiovascular $(21,6 \%)$, doença pulmonar $(17,6 \%)$, neoplasia $(12 \%)$ e acidente vascular cerebral $(12 \%)$ ente outras enfermidades que representaram 36,8\% do diagnóstico clínico.

Quanto ao IMC, a média encontrada entre os pacientes avaliados foi de $21,4 \pm 6,4 \mathrm{~kg} / \mathrm{m}^{2}$, não havendo diferença entre os sexos. A distribuição do diagnóstico nutricional, segundo este dado, foi de $54,2 \%$ dos pacientes com baixo peso, 32,2\% eutróficos e $13,6 \%$ apresentavam excesso de peso.

\section{Tabela 1}

Perfil geral dos pacientes avaliados

\begin{tabular}{lrcc}
\hline & Sexo feminino $(\mathrm{n}=30) \mathrm{X} \pm \mathrm{DP}$ & Sexo masculino $(\mathrm{n}=29) \mathrm{X} \pm \mathrm{DP}$ & Total $(\mathrm{n}=59) \mathrm{X} \pm \mathrm{DP}$ \\
\hline Idade $(\mathrm{anos})$ & $71,56 \pm 17,77$ & $67,00 \pm 12,84$ & $69,32 \pm 15,58$ \\
Peso $(\mathrm{kg})$ & $52,59 \pm 0,07$ & $61,46 \pm 15,19$ & $59,95 \pm 15,81$ \\
Estatura $(\mathrm{m})$ & $1,57 \pm 0,07$ & $1,68 \pm 0,09$ & $1,63 \pm 0,10$ \\
$\mathrm{IMC}\left(\mathrm{kg} / \mathrm{m}^{2}\right)$ & $20,71 \pm 5,99$ & $22,26 \pm 6,99$ & $21,47 \pm 6,49$ \\
$\mathrm{CB}(\mathrm{cm})$ & $26,75 \pm 5,72$ & $26,72 \pm 4,46$ & $16,73 \pm 5,10$ \\
DCT $(\mathrm{mm})$ & $15,80 \pm 7,44$ & $12,86 \pm 7,74$ & $14,35 \pm 7,67$ \\
CMB $(\mathrm{cm})$ & $21,62 \pm 4,01$ & $22,46 \pm 3,43$ & $22,03 \pm 7,73$ \\
GET $(\mathrm{kcal} /$ dia $)$ & $1458,42 \pm 314,19$ & $1818,94 \pm 435,49$ & $1635,63 \pm 417,08$ \\
\hline
\end{tabular}

IMC: índice de massa corporal. CB: circunferência braquial. DCT: dobra cutânea tricipital. CMB: circunferência muscular do braço. GET: gasto energético total. 
Para a determinação do perfil nutricional, foram avaliados peso, estatura, circunferências braquial (CB) e muscular do braço (CMB), dobra cutânea triciptal (DCT) e índice de massa corpórea (IMC) (Tabela 2). Os dados da DCT e CMB apresentaram diferença entre os sexos $(\mathrm{p}<0,05)$ e nos sugerem maior comprometimento da massa muscular em relação ao tecido adiposo, fato possivelmente relacionado à perda de peso não intencional associada à doença, a imobilidade e à média de idade (69 anos) da população deste estudo.

Em relação à via de acesso, $83 \%$ receberam nutrição enteral (NE) por via nasogástrica (NSG), $10 \%$ por via nasoduodenal (NSE) e 7\% por jejunostomia $(\mathrm{JN})$, sendo o método intermitente gravitacional empregado em todos os pacientes para a administração da NE.

Para cada paciente avaliado foi verificada a prescrição dietética da TNE em relação a calorias e comparada com as reais necessidades energéticas estimadas e com a energia efetivamente ofertada.

A necessidade energética (GET) média foi de $1635 \pm 417,8 \mathrm{Kcal} / \mathrm{dia}$. A média de energia prescrita foi de $1045 \mathrm{kcal} /$ dia e a ofertada, a partir do quarto dia de implementação da TNE, foi de $1035 \pm 422 \mathrm{Kcal} /$ dia. Verificou-se uma diferença significativa entre a necessidade e a oferta energética por meio da NE $(\mathrm{p}<0,05)$ assim como entre ao valor energético necessário e o prescrito $(\mathrm{p}<0,05)$, ficando ambos aquém da necessidade (Tabela 3). Não foi observada diferença significativa entre os sexos, a via de administração e o diagnóstico nutricional com base no IMC.

$\mathrm{O}$ volume médio prescrito de NE para os pacientes avaliados foi de $950 \pm 302 \mathrm{~mL} / \mathrm{dia}$. Considerando a densidade energética das fórmulas enterais administradas nestes pacientes, estimou-se que o volume médio necessário de NE para suprir as necessidades energéticas diárias dos pacientes avaliados seria

\section{Tabela 2}

Frequência (\%) da adequação nutricional dos indicadores IMC, DCT e CMB dos pacientes avaliados

\begin{tabular}{|c|c|c|c|c|c|c|c|c|c|}
\hline \multirow{2}{*}{$\begin{array}{l}\text { Adequação } \\
\text { nutricional }\end{array}$} & \multicolumn{3}{|c|}{ IMC } & \multicolumn{3}{|c|}{ DCT } & \multicolumn{3}{|c|}{ CMB } \\
\hline & $\begin{array}{c}\text { Feminino } \\
n=30\end{array}$ & $\begin{array}{c}\text { Masculino } \\
n=29\end{array}$ & $\begin{array}{l}\text { Total } \\
n=59\end{array}$ & $\begin{array}{c}\text { Feminino } \\
\mathrm{n}=30\end{array}$ & $\begin{array}{c}\text { Masculino } \\
\mathrm{n}=29\end{array}$ & $\begin{array}{l}\text { Total } \\
\mathrm{n}=59\end{array}$ & $\begin{array}{c}\text { Feminino } \\
\mathrm{n}=30\end{array}$ & $\begin{array}{c}\text { Masculino } \\
\mathrm{n}=29\end{array}$ & $\begin{array}{l}\text { Total } \\
\mathrm{n}=59\end{array}$ \\
\hline Abaixo do normal & 56,7 & 51,7 & 54,2 & $53,3^{\mathrm{a}}$ & $27,6^{\mathrm{b}}$ & 40,7 & $36,7^{\mathrm{a}}$ & $75,9^{\mathrm{b}}$ & 55,9 \\
\hline Normal & 30,0 & 34,5 & 32,2 & $43,3 \mathrm{a}$ & $44,8 b$ & 44,1 & $46,6^{\mathrm{a}}$ & $24,1^{\mathrm{b}}$ & 35,6 \\
\hline Acima do normal & 13,3 & 13,8 & 13,6 & $3,4^{\mathrm{a}}$ & $27,6^{\mathrm{b}}$ & 15,2 & $16,7^{\mathrm{a}}$ & $0,0^{\mathrm{b}}$ & 8,5 \\
\hline Total & 100,0 & 100,0 & 100,0 & 100,0 & 100,0 & 100,0 & 100,0 & 100,0 & 100,0 \\
\hline
\end{tabular}

Os valores, na mesma linha, sobrelevados de letras diferentes, diferem estatisticamente entre os sexos, pelo teste $t$ de Student a 5\% de probabilidade.

Tabela 3

Parâmetros da TNE segundo o diagnóstico nutricional

\begin{tabular}{lccc}
\hline & Baixo Peso & Eutrofia & Excesso de Peso \\
& $\mathrm{N}=32$ & $\mathrm{~N}=19$ & $\mathrm{~N}=08$ \\
\hline Necessidade energética - GET (kcal/dia) & $1513,08^{\mathrm{a}}( \pm 331,97)$ & $1666,41^{\mathrm{b}}( \pm 385,09)$ & $1970,52^{\mathrm{c}}( \pm 597,48)$ \\
Oferta energética (kcal/dia) & $1124,00^{\mathrm{a}}( \pm 356,0)$ & $963,73^{\mathrm{a}}( \pm 404,64)$ & $939,56^{\mathrm{a}}( \pm 539,59)$ \\
Volume de NE necessário $(\mathrm{mL})$ & $1368,5^{\mathrm{a}}( \pm 299,51)$ & $1558,16^{\mathrm{b}}( \pm 350,1)$ & $1791,4^{\mathrm{c}}( \pm 543,2)$ \\
Volume de NE prescrito $(\mathrm{mL})$ & $974,01^{\mathrm{a}}( \pm 279,24)$ & $872,25^{\mathrm{a}}( \pm 296,96)$ & $1042,5^{\mathrm{a}}( \pm 467,08)$ \\
Volume de NE administrado $(\mathrm{mL})$ & $913,51^{\mathrm{a}}( \pm 288,36)$ & $791,24^{\mathrm{a}}( \pm 329,18)$ & $892,5^{\mathrm{a}}( \pm 382,91)$ \\
\hline
\end{tabular}

NE: nutrição enteral.

Os valores, na mesma linha, sobrelevados de letras diferentes, diferem estatisticamente entre os grupos, pelo teste $t$ de Student a $5 \%$ de probabilidade. 
de aproximadamente $1500 \pm 319 \mathrm{~mL} / \mathrm{dia}$. Assim observamos uma diferença significante $(\mathrm{p}<0,05)$ entre $\mathrm{o}$ volume prescrito e o realmente necessário para suprir a necessidade energética. A média diária do volume de NE administrado foi de $869,2 \pm 313 \mathrm{~mL} / \mathrm{dia}$, o qual não apresentou diferença estatística $(\mathrm{p}>0,05)$ em relação ao prescrito, mas sim em relação ao volume de NE necessário $(\mathrm{p}<0,05)$.

O déficit de volume administrado em relação ao prescrito, neste estudo foi associado à suspensão de horários de administração. No hospital em que o estudo foi realizado, no método intermitente são previsto 8 horários de administração da NE. Entretanto, nos pacientes avaliados houve, em média, suspensão de 1,6 horários ao dia. As intercorrências informadas nas evoluções de enfermagem como justificativa para a suspensão de horários de administração de NE foram: a ocorrência de refluxo (15\%), diarréia (15\%), resíduo gástrico $(7,5 \%)$, distensão abdominal $(2,5 \%)$ e aspiração pulmonar $(2,5 \%)$ entre outras que incluíram jejum para realização de exame, deslocamento do paciente para outras unidades, pausa para procedimentos de rotina, atraso na infusão do horário anterior e deslocamento da sonda.

\section{Discussão}

Quanto ao estado nutricional, a maioria (54,2\%) dos pacientes apresentava baixo peso segundo o IMC, resultados semelhantes ao estudo de Campos et al. ${ }^{22}$ que encontraram 54,8\% de desnutrição entre os pacientes com TNE avaliados. Ao contrário, Nozaki et al. ${ }^{23}$ encontraram maior prevalência $(57 \%)$ de pacientes eutróficos segundo este dado.

A necessidade energética média dos pacientes avaliados foi de $1642 \mathrm{kcal} / \mathrm{dia}$, enquanto que a ofertada por meio da TNE foi de $1035 \mathrm{kcal} / \mathrm{dia}$, indicando 63\% de adequação energética.

Diferentes estudos têm encontrado adequações energéticas administradas e prescritas inferiores a $90 \%$, considerado este, como valor de referência padrão para comparação através da relação percentual ${ }^{24,25}$.

Broek et al. ${ }^{26}$ ao analisarem a porcentagem de adequação de energia de pacientes com NE exclusiva, obtiveram uma média de $87 \%$ de adequação do administrado frente ao prescrito. Um outro estudo desenvolvido por De Jonghe et al. ${ }^{27}$ com 51 pacientes de UTI em TNE, obteve uma média de necessidades energéticas de $28,1 \pm 4,7 \mathrm{kcal} / \mathrm{kg} / \mathrm{dia}$, sendo que a prescrita foi de $22 \pm 8,6 \mathrm{kcal} / \mathrm{kg} / \mathrm{dia}$ (78,2\% das necessida- des diárias) e a efetivamente consumida foi de $20,0 \pm 7,9 \mathrm{kcal}$ ( $63,5 \%$ das necessidades diárias).

No presente estudo, a inadequação energética teve início na precrição dietética da TNE, considerando que o do volume de NE prescrito ficou abaixo do volume necessário para atender a demanda energética dos pacientes. O volume médio de NE prescrito levaria a uma oferta energética média de $1045 \mathrm{kcal} /$ dia, a qual representa $64 \%$ da necessidade energética dos pacientes avaliados (1642 kcal/dia). Em vários estudos $^{7,23,28}$ verificou-se que, em média, a energia prescrita pela equipe foi menor que a necessidade energética estimada.

Adam e Batson ${ }^{29}$ realizaram a mesma análise em cinco UTIs da Inglaterra e observaram que a energia prescrita atendia entre $76 \%$ e $100 \%$ da necessidade. Igualmente ao presente estudo, Poltronieri ${ }^{30}$ detectou que tanto o volume prescrito quanto o administrado foram, em média, menores que o volume necessário para suprir a necessidade energética.

No presente estudo, pode-se observar que o déficit de energia prescrita em relação a necessária ocorreu principalmente pelo fato de não haver na rotina desse hospital a prática do cálculo da necessidade energética individual com uso de equações preditivas baseadas nos dados de cada paciente, como sexo, idade, peso e estatura. A falta de profissionais capacitados para o grande número de pacientes em uso de TNE também pode ter contribuído para este fato. Por outro lado, deve-se ter muita cautela ao se estimar o GEB por equações de predição, uma vez que podem fornecer estimativas elevadas do GEB acarretando erros na estimativa da necessidade energética de indivíduos. ${ }^{31}$

Teixeira et al. ${ }^{14}$ comparando a oferta energética prescrita com a administrada, encontraram adequação de $74,4 \%$, semelhante ao resultado obtido por De Jonghe et al. ${ }^{27}$ que foi de $71 \%$ de adequação. Engel et al. ${ }^{32}$ observaram que apenas $35 \%$ dos indivíduos avaliados recebiam a meta de $80 \%$ das necessidades energéticas reais.

Cartolano et al. ${ }^{33}$ realizaram, por quatro anos consecutivos (2005 a 2008), levantamento dos valores médios de energia e proteínas calculados, prescritos e realmente administrados a pacientes adultos com TNE internados na unidade de terapia intensiva de um hospital universitário da cidade de São Paulo. A meta energética prescrita foi muito próxima da meta energética calculada, permanecendo próxima a $100 \%$ de adequação em todos os levantamentos. Já a adequação percentual entre energia administrada e prescrita ficou 
abaixo de $90 \%$ em todos os anos. Porém é importante salientar que no decorrer dos quatro anos houve uma positiva aproximação entre meta nutricional prescrita e os valores de energia realmente infundidos que aumentou de 74\% em 2005 para 89\% em 2008. Os autores atribuíram tal resultado a presença de protocolo de infusão de NE previamente estabelecido e presença de equipe multiprofissional de terapia nutricional, bem como a educação continuada da equipe.

No presente estudo a porcentagem de adequação da necessidade energética real em relação à administrada por meio da TNE foi de $64 \%$ caracterizando um déficit energético maior do que os relatados na literatura. Diferentemente, Fernandes et al. ${ }^{34}$ não encontraram diferença estatística entre os valores de energia prescritos pela equipe de nutricionistas do local e os valores realmente ofertados aos pacientes, apesar de observarem intercorrências que determinaram a diminuição da oferta energética total aos pacientes. Oliveira et al. ${ }^{35}$ avaliaram 77 pacientes em UTI com uso de TNE exclusiva e também não observaram inadequação quando compararam a necessidade energética com a energia efetivamente oferecida visto que, em média, a adequação encontrada foi de $94,5 \%$, apesar da elevada ocorrência de complicações gastrointestinais, sendo o refluxo gástrico o mais prevalente (39\%).

A inadequação energética, no atual estudo, foi marcada pelo déficit do volume de NE administrado (média de 869,2 mL/dia) o qual ficou abaixo do prescrito (média de $950 \mathrm{~mL} /$ dia) e do necessário para atender a necessidade energética (média de $1500 \mathrm{~mL} /$ dia).

No hospital onde foi realizado este estudo adota-se a frequência de 8 horários diários de administração da NE no método intermitente gravitacional, adotado em todos os pacientes deste estudo. Entretanto, foi observado a suspensão de cerca de 1,6 horários de administração ao dia, fato que culminou no déficit do volume administrado em relação ao prescrito. Refluxo, diarréia e resíduo gástrico configuraram as principais justificativas para as suspensões de horários de infusão.
Couto et al. ${ }^{12}$ referiram em seu estudo que as complicações mais frequentes que levaram à suspensão da NE foram à estase gástrica, interrupção na dieta para procedimentos da enfermagem e da fisioterapia, jejum para procedimentos e obstrução ou deslocamento da sonda. Entretanto, Mc Clave et al. ${ }^{36}$ constataram que em $66 \%$ dos casos de intercorrências houve carência de educação e conhecimento por parte da equipe responsável pelo tratamento dos pacientes.

A desnutrição é frequentemente observada em pacientes hospitalizados ${ }^{37,38,39}$ e está relacionada tanto com as doenças como com a prescrição dietética aplicada. $^{23}$

O suporte nutricional oferecido ao paciente tem por objetivo evitar a perda de proteínas viscerais e musculares, além de prover energia e substratos suficientes à manutenção do estado fisiológico ${ }^{40}$. O paciente hospitalizado, frequentemente, se encontra hipermetabólico decorrente do seu estado patológico. Este fato associado ao déficit energético constatado neste estudo possivelmente tenha participado da gênese ou agravo do comprometimento nutricional, considerando que $54,2 \%$ dos pacientes deste estudo apresentaram IMC abaixo do normal.

\section{Conclusão}

Os resultados deste estudo indicam que tanto a prescrição como a oferta energética não foram baseadas nas necessidades destes pacientes, acarretando um déficit energético importante, que pode levar ao agravo do estado nutricional dos mesmos. Em pacientes hospitalizados em uso de TNE, a prescrição energética deve ser feita pelo profissional habilitado dentro da equipe multidisciplinar, tendo como base as necessidades de cada paciente.

Os resultados deste estudo nos mostram a necessidade de adoção de mecanismos de vigilância clínica afim de assegurar que um melhor manejo nutricional seja instituído a pacientes hospitalizados em uso de TNE. 


\begin{abstract}
Objective: evaluating the adequacy, prescription and energy supply of enteral nutrition therapy in hospitalized patients. Methods: was performed a retrospective survey of the evolution of TNE protocol of 59 patients hospitalized in a general hospital in Marília / SP / BR. Data collected included gender, age, clinical diagnosis, anthropometric and dietary data related to the prescribed dietary formula, route and method of administration of the enteral nutrition, the daily volume prescribed and administered, the daily amount of energy required and offered in the diet, as well as possible complications. Body mass index (BMI), triceps skinfold (TSF) and arm muscle circumference (AMC) were used to establish the nutritional status of the patients. The adequacy of ENT was done according to the daily energy requirement compared to the average energy received during the daily use of ENT. Results: The average found to the BMI was $21.4 \mathrm{~kg} / \mathrm{m}^{2}$, and no differences were found comparing male and female. The data for TSF and AMC were different between genders $(p<0.05)$ and suggest a more severe muscle mass in relation to adipose tissue. The averaged to the energy requirements was $1642 \mathrm{kcal} /$ day but the average of energy prescribed was $1045 \mathrm{kcal} /$ day and the amount offered was $1035 \mathrm{kcal} /$ day. There was a significant difference between the necessity and the supplied energy offered through enteral nutrition $(p=0.00)$ as well between the energy required and prescribed $(p=0.00)$, both were shorter than the necessity. There was no significant difference ( $p>0.05)$ in energy supply, volume of enteral nutrition prescribed and administered among eutrophic, underweight or overweight patients. Conclusion: The results of this study indicate that the prescription and energy supply were not based on the needs of these patients, causing a significant energy deficit, which can lead to worsening of the nutritional status of the same. In hospitalized patients in use of ENT, the energy requirement must be made by a qualified professional within the multidisciplinary team, based on the needs of each patient.
\end{abstract}

Key words: Nutrition therapy. Enteral nutrition. Nutritional status. Nutritional requirements. Hospitalization.

\section{Referências bibliográficas}

1. Brasil. Ministério da Saúde. Agência Nacional de Vigilância Sanitária. Portaria oㅜ 337, de 14 de abril de 1999. Regulamento técnico para fixar os requisitos mínimos exigidos para a terapia de nutrição enteral. Diário Oficial da União. 15 abr 1999.

2. Peter JV, Moran JL, Phillips-Hughes J. A metaanalysis of treatment outcomes of early enteral versus early parenteral nutrition in hospitalized patients. Crit Care Med. 2005; 33: 213-20.

3. Baxter YC, Waitzberg DL. Alimentação enteral. In: Silva SMCS, Mura JDP. Tratado de alimentação, nutrição e dietoterapia. São Paulo: Roca; 2007. p. 873-82.

4. Waitzberg DL, Fabul RA, Aanholt DPJV, Plopper C, Terra RM. Indicações e técnicas de ministração em nutrição enteral. In: Waitzberg DL. Nutrição oral, enteral e parenteral na prática clínica. 3를 ed. São Paulo: Atheneu; 2006. p. 561-71.

5. David CM. Terapia nutricional no paciente grave. Rio de Janeiro: Revinter; 2001.

6. Schieferdecker MEM. Estado Nutricional de pacientes em terapia nutricional enteral e a relação das necessidades energéticas com o valor energético total prescrito e recebido [Dissertação de mestrado]. Curitiba: Universidade Federal do Paraná; 2005.

7. Campanella LCA, Silveira BM, Neto OR, Silva AA. Terapia nutricional enteral: a dieta prescrita é realmente infundida? Rev Bras Nutr Clín. 2008; 23: 21-5.

8. Correia MITD. Repercussões da desnutrição sobre a morbimortalidade e custos em pacientes hospitalizados no Brasil [Tese de Doutorado], São Paulo: Faculdade de Medicina USP; 2000.
9. Villet S, Chiolero RL, Bollman MD, Revelly JP, Cayeux RN, Delarue J, Berger MM. Negative impact of hypocaloric feeding and energy balance on clinical outcome in ICU patients. Clin Nutr. 2005; 24: 502-9.

10. Cabrera LS, Navarro GO, Martul MG, Rodrígues AR, Palacios MS, Medicina EH. Calidad del soporte nutritional artificial en una unidad de cuidados intensivos. Nutr Hosp. 2006; 21:6616.

11. Petros S, Engelmann L. Enteral nutrition delivery and energy expenditure in medical intensive care patients. Clin Nutr. 2006; 25:51-9.

12. Couto JCF, Bento A, Couto CMF, Silva BCO, Oliveira IAG. Nutrição enteral em terapia intensiva: o paciente recebe 0 que prescrevemos? Rev Bras Nutr Clín. 2002; 17: 43-6.

13. O'Meara D, Mireles-Cabodevila E, Frame F, Hummell AC, Hammel J, Dweik RA, Arroliga AC. Evaluation of delivery of enteral nutrition in critically ill patients receiving mechanical ventilation. Am J Crit Care. 2008; 17:53-61.

14. Teixeira AC de C, Caruso L, Soriano FG. Terapia Nutricional Enteral em Unidade de Terapia Intensiva: Infusão Versus Necessidades. Rev Bras Ter Intensiva. 2006; 18: 331-7.

15. Elpern EH; Stutz L; Peterson $S$ et al. Outcomes associated with enteral tube feedings in a medical intensive Care Unit. Am J Crit Care. 2004; 13:221-7.

16. World Health Organization. Obesity: preventing and managing the global epidemic. In: World Health Organization. Geneva; 1998. (WHO Technical Report Series, 894).

17. Lipschitz DA. Screening for nutritional status in the elderly. Prim Care. 1994; 21: 55-67.

18. Frisancho AR. Anthropometric standards for the assessment of growth and nutritional status. University of Michigan Press, 1990. 
19. Blackburn GL, Thornton PA. Nutritional assessment of the hospitalized patient. Med Clin North America. 1979, 63:1110315.

20. Harris JA, Benedict FG. Biometric studies of basal metabolism in man. Washington, DC: Carnegie Institute of Washington, 1919.

21. Long CL, Schaffel N, Geiger JW, Schiller WR, Blakemore WS. Metabolic response to injury and illness: estimation of energy and protein needs from indirect calorimetry and nitrogen alance. J Parenter Enteral Nutr. 1979; 3:452-6.

22. Campos DJ, Silva AFF, Souza MHS, Shieferdecker ME. Otimização do fornecimento calórico-protéico na de terapia nutricional enteral em unidade de terapia intensiva com o uso de protocolo. Rev Bras Nutr Clín. 2006; 21: 2-5.

23. Nozaki VT; Peralta RM; Fernandes CAM. Terapia nutricional enteral: análise dos requerimentos energéticos e perfil nutricional. Rev Bras Nutr Clín. 2009; 29(3): 143-8.

24. Binnekade JM, Tepaske R, Bruynzeel P, Mathus-Vliegen EM, Hann RJ. Daily enteral feeding practice on the ICU: attainment of goals and interfering factors. Crit Care. 2005; 9: 218-25.

25. O'Leary-Kelley CM, Puntilho KA, Barr J, Stotts N, Douglas MK. Nutritional adequacy in patients receiving mechanical ventilation who are fed enterally. Am J Crit Care. 2005; 14 : 222-31.

26. Broek PWV, Rasmussen-Conrad EL, Naber AH, Wanten GJ. What you think is not what they get : significant discrepancies between prescribed and administered doses of tube feeding. Br J Nutr. 2009; 101: 68-71.

27. De Jonghe B, Appere-De-Vechi C, Fournier M, Tran B, Merrer $\mathrm{J}$, Melchior JC, et al. A prospective survey of nutritional support practices in intensive care unit patients: what is prescribed? What is delivered? Crit Care Med. 2001; 29: 8-12.

28. Luft VM; Vieira DM; Beghetto MG; Polanczyk CA; Mello ED. Suprimento de micronutrientes, adequação energética e progressão da dieta enteral em adultos hospitalizados. Rev Nutr. 2008; 21: 513-23.

29. Adam S, Batson S. A study of problems associated with the delivery of enteral feed in critically ill patients in five ICUs in the UK. Intensive Care Med. 1997; 23: 261-6.
30. Poltronieri MF de A. Eventos adversos na administração de dieta enteral em unidade de terapia intensiva: análise comparativa entre o volume prescrito e o administrado. [Dissertação de Mestrado]. São Paulo: Escola de Enfermagem da Universidade de São Paulo - USP; 2006.

31. Wahrlich V, Anjos LA. Validação de equações de predição da taxa metabólica basal em mulheres residentes em Porto Alegre, RS, Brasil. Rev Saúde Pública. 2001; 35: 39-45.

32. Engel JM, Muhling J, Junger A, Menges T, Karcher B, Hempelmann $G$. Enteral nutrition practice in a surgical intensive care unit: what proportion of energy expenditure is delivered enterally? Clin Nutr. 2003; 22: 187-92.

33. Cartolano FC; Caruso L; Soriano FG. Terapia nutricional enteral: aplicação de indicadores de qualidade. Rev Bras Ter Intensiva. 2009, 21: 376-83.

34. Fernandes DD, Ghisleni DR, Colpo E, Lopes LFD, Rubin BA. Aporte nutricional em pacientes em terapia enteral exclusiva: recomendado x recebido. Rev Bras Nutr Clín. 2009; 24: 8592.

35. Oliveira SM, Burgos MGPA, Santos EMC, Prado LVS, Petribú MMV, Bomfim MTS. Complicações gastrointestinais e adequação calórico-protéica de pacientes em uso de nutrição enteral em uma unidade de terapia intensiva. Rev Bras Ter Intensiva. 2010; 22: 270-3.

36. Mc Clave SA, Sexton LK, Spain DA, Adams JL, Owens NA, Sullins $M B$, et al. Enteral tube feeding in the intensive care unit: factors impending adequate delivery. Crit Care Med. 1999; 27: 1252-6.

37. Waitzberg DL, Caiaffa WT, Correia MI. Hospital malnutrition: the Brazilian national survey (IBRANUTRI): a study of 4000 patients. Nutrition. 2001; 17(7-8): 573-80.

38. Mías C, Jürschik P, Massoni T, Sadurní M, Aquilà JJ, Solá R, et al. Evaluación del estado nutricional de los pacientes mayores atendidos em una unidad de hospitalización a domicilio. Nutr Hosp. 2003; 18: 6-14.

39. Pirlich M, Schütz T, Kemps M, Luhman N, Minko N, Lübke HJ, et al. Social risk factors for hospital malnutrition. Nutrition. 2005; 21: 295-300.

40. Lemos CFS, Paula CA, Rocha R. Alterações gastrintestinais de pacientes críticos em uso de norepinefrina e terapia nutricional enteral. Rev Bras Nutr Clin. 2008; 23: 34-40. 